\title{
Madurez sexual e indicadores asociados en dos genotipos de gallinas bajo diferentes programas de alimentación
}

\author{
Sanz, P. ; Sindik, M. ${ }^{1,2}$; Fernández, R. ${ }^{1}$; Revidatti, F. ${ }^{1}$ \\ ${ }^{1}$ Cátedra Producción de Aves, Fac. Cs. Vet. UNNE, Cabral 2139, 3400, Corrientes (Argentina).

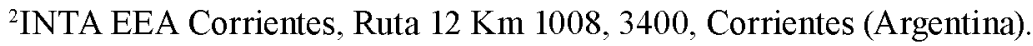 \\ E-mail: granja@vet.unne.edu.ar
}

\begin{abstract}
Resumen
Sanz, P.; Sindik, M.; Fernández, R.; Revidatti, F.: Madurez sexual e indicadores asociados en dos genotipos de gallinas bajo diferentes programas de alimentación. Rev. Vet. 31: 2, 142-145, 2020. El objetivo del presente estudio fue evaluar el peso, la condición corporal, los indicadores zoométricos y la edad a la madurez sexual en dos genotipos de gallinas reproductoras Campero INTA, bajo dos programas de alimentación en cría y recría. Se trabajó con 400 gallinas pertenecientes a 2 genotipos (sintética $\mathrm{E}$ y el híbrido $\mathrm{ES} * \mathrm{~A}$ ) que recibieron dos planes nutricionales (control y tratado) entre las semanas 5 y 22 . A las 22 semanas se observó una interacción estadísticamente significativa $(p<0,0001)$ para el peso corporal. En el grupo control las aves del genotipo $\mathrm{ES}^{*} \mathrm{~A}$ fueron más pesadas que la población $\mathrm{E}$ ( $\mathrm{ES} * \mathrm{~A}$ $2.288 \mathrm{~g}$ vs $\mathrm{E} 2.133 \mathrm{~g}$ ), mientras que en el grupo tratado las aves del genotipo $\mathrm{E}$ fueron más pesadas que la población ES*A (ES*A $2.313 \mathrm{~g}$ vs E $2.348 \mathrm{~g}$ ). El índice de cresta registró diferencias significativas entre genotipos $(\mathbf{p}<0,0001)$ y programas de alimentación $(\mathbf{p}<0,0001)$. El programa de alimentación indujo un adelantamiento de la entrada a la madurez sexual en días para ambas poblaciones ( $\mathrm{p}=0,009$ ), con valores de $165 \pm 2,83$ ( $\mathrm{ES} * \mathrm{~A}$ Control), $156 \pm 2,83$ (ES*A Tratado), 164 $\pm 0,00$ (E Control) y 157 $\pm 2,83$ (E Tratado). Se concluye que diferentes asignaciones de alimento durante las etapas de cría y recría impactan sobre el peso corporal y los indicadores zoométricos en los genotipos maternos del pollo Campero INTA y sus cruzamientos, con diferencias marcadas en el grado de integración entre peso corporal y desarrollo reproductivo entre ambos genotipos. La mayor asignación produce un adelantamiento a la entrada de la madurez sexual.
\end{abstract}

Palabras clave: gallinas, alimentación, reproducción, manejo, restricción.

\begin{abstract}
Sanz, P.; Sindik, M.; Fernández, R.; Revidatti, F.: Sexual maturity and associated indicators in two genotypes of hens under two feeding programs. Rev. Vet. 31: 2, 142-145, 2020. The aim of the present study was to evaluate body weight, corporal condition, zoometric indicators and age at sexual maturity in two genotypes of Campero INTA breeders under two raising feeding programs. We worked with 400 pullets from 2 genotypes (synthetic $\mathrm{E}$ and the hybrid $E S^{*} \mathrm{~A}$ ) that received two nutritional levels (control and treated) between weeks 5 and 22 . At 22 weeks a statistically significant interaction was observed $(\mathbf{p}<0,0001)$ for body weight. In the control group the birds of the ES*A genoty pe were heavier than the population E (ES*A 2,288 g vs. E 2,133 g), while in the treated group the birds of the genoty pe E were heavier than the population ES*A (ES*A 2,313 g vs. E 2,348 g). The comb index recorded significant differences between genotypes $(p<0.0001)$ and feeding programs $(p<0.0001)$. The feeding program induced an advance of the onset to sexual maturity in days for both populations ( $\mathrm{p}=0.009$ ), with values of $165 \pm 2.83$ (ES*A Control), $156 \pm 2.83$ (ES*A Treated), $164 \pm$ 0.00 (E Control) and $157 \pm 2.83$ (E Treated). We concluded that different food assignments during the raising period impact on the body weight and the zoometric indicators in the maternal genoty pes of the Campero INTA chicken and its crossings, with marked differences in the degree of integration between body weight and reproductive development between both genoty pes. The higher allocation produces an advance at the entrance of sexual maturity.
\end{abstract}

Key words: hens, poultry, reproduction, management, restriction. 


\section{INTRODUCCIÓN}

En la gallina doméstica la selección genética para producción de carne dio por resultado correlaciones que expresan la magnitud en la cual dos caracteres están asociados genéticamente, en tanto que el ambiente es una causa de asociación cuando dos caracteres están influidos por las mismas condiciones ambientales ${ }^{8}$.

La selección llevó a la obtención de líneas pesadas para producción de carne que presentan una marcada correlación negativa entre aptitud reproductiva y peso corporal, lo cual hace necesario desarrollar estrategias de manejo efectivas, que permitan adecuar las señales ambientales a los objetivos específicos de la reproducción.

Entre estas, la alimentación se destaca por su repercusión sobre la producción de hormonas y su efecto sobre el desarrollo folicular, tanto durante las etapas previas a la madurez sexual como en el ave adulta.

La falta de ajuste en los programas provoca disrupciones en el patrón normal de producción o secreción de la hormona liberadora de gonadotropina y las condiciones en que se desarrolla la madurez sexual ${ }^{2}$.

La madurez sexual en las gallinas es un proceso biológico complejo, lo que implica que se desarrolla en determinado momento de la vida y bajo ciertas circunstancias específicas. Se ha señalado que la madurez sexual es el resultado combinado de varios factores que incluyen la edad cronológica, el peso y la composición corporal del ave ${ }^{9}$.

Durante la maduración sexual los cambios neuroendocrinos y metabólicos producen transformaciones en el aparato reproductor y estimulan el desarrollo de caracteres sexuales secundarios ${ }^{5}$, de ahí que, para evaluar el grado de desarrollo genital alcanzado por las reproductoras al momento de la madurez sexual y la evolución del mismo a lo largo de la etapa de postura, existan un amplio grupo de técnicas que pueden ser utilizadas.

Hacia el final de la recría, la cresta aumenta de volumen, se torna turgente y el ave puede perder algunas plumas primarias (muda prenupcial) y los huesos púbicos se ensanchan para dar paso al huevo ${ }^{6}$.

La primera oviposición es considerada convencionalmente como el inicio de la madurez sexual, sin embargo en los programas de investigación sobre fisiología reproductiva se requiere establecer en forma más precisa el momento en que este fenómeno se produce.

El objetivo del presente estudio fue evaluar el peso, la condición corporal, los indicadores zoométricos y la edad a la madurez sexual en dos genotipos de gallinas reproductoras camperas, bajo dos programas de alimentación en cría y recría.

\section{MATERIAL Y MÉTODOS}

Las distintas etapas del ciclo de los reproductores se llevaron a cabo en el Centro de Multiplicación de Aves de la Estación Experimental Agropecuaria Co- rrientes del INTA, ubicada en la Ruta Nacional 12, km 1008, El Sombrero (Corrientes), Argentina.

En el ensayo se trabajó con 200 gallinas pertenecientes a la población sintética materna E del pollo Campero INTA (composición genética teórica 50\% Cornish Colorada; $50 \%$ Rhode Island Colorada) y 200 pertenecientes al híbrido simple producto del cruzamiento entre las poblaciones sintéticas maternas ES*A (composición genética teórica $81,25 \%$ Cornish Colorada; $18,75 \%$ Rhode Island Colorada).

Las aves de cada genotipo se subdividieron en dos grupos, cada uno de los cuales recibió entre las semanas 5 y 22 uno de los siguientes tratamientos que consistieron en modificaciones cuantitativas de las dietas: grupo estándar (programa de alimentación restringida habitualmente aplicado en el establecimiento) y grupo stándar +10 (programa de alimentación con una modificación cuantitativa de la dieta consistente en un aumento del $10 \%$ en el aporte de nutrientes respecto del grupo control).

En las semanas 22 y 24 del ciclo de las reproductoras se registró individualmente en cada ave: el peso corporal (PC), condición corporal (CC, escala ${ }^{4}$ de Gregory y Robins 0 a 3), índice de cresta (IC), producto del alto de cresta desde su base hasta la punta de la cuarta espiga por su longitud desde el extremo anterior hasta el posterior, ambos registrados con calibre en $\mathrm{mm}^{2}$, ancho de cloaca $(\mathrm{ACl}$, distancia transversal en mm existente entre ambos extremos laterales de la cloaca con el ave en decúbito dorsal), ancho de la cadera (ACa, distancia transversal en mm existente entre ambos isquiones con el ave en decúbito dorsal) y longitud abdominal (LAb, distancia longitudinal en mm existente entre la apófisis esternal caudal y una línea imaginaria que une ambos extremos de los huesos coxales con el ave en decúbito dorsal).

Se registró la edad a la madurez sexual (MS, en días) por observación directa, tomando como criterio la puesta del primer huevo en cada box. El análisis comparativo se efectuó mediante un diseño experimental factorial $2 \times 2$ (dos genotipos y dos planos nutricionales) considerando límite un nivel de significancia de $5 \%{ }^{11} \mathrm{e}$ incluyendo en el modelo la interacción entre los factores sometidos a estudio.

El efecto del genotipo sobre la proporción de aves en las diferentes categorías de condición corporal, se evaluó con una prueba Chi-cuadrado de homogeneidad, y su efecto sobre el peso corporal con un análisis de la variancia a un criterio de clasificación seguido del Test de Duncan.

\section{RESULTADOS Y DISCUSIÓN}

En las Tablas 1 y 2 se exponen los resultados obtenidos al final de la recría (22 semanas) para los distintos grupos experimentales y su significancia estadística.

El peso corporal demostró diferencias significativas entre ambos genotipos y programas de alimentación aplicados durante las etapas de cría y recría, ob- 
Tabla 1. Peso corporal e indicadores zoométricos de madurez sexual en dos genotipos de reproductoras Campero INTA bajo dos regímenes de asignación de nutrientes.

\begin{tabular}{lcccc}
\hline \multirow{2}{*}{ variables } & \multicolumn{2}{c}{ híbridas ES*A } & \multicolumn{2}{c}{ sintéticas E } \\
\cline { 2 - 5 } & $\begin{array}{c}\text { estándar } \\
\overline{\mathrm{x}} \pm \mathrm{EE}\end{array}$ & $\begin{array}{c}\text { estándar 10\% } \\
\overline{\mathrm{x}} \pm \mathrm{EE}\end{array}$ & $\begin{array}{c}\text { estándar } \\
\overline{\mathrm{x}}+\mathrm{EE}\end{array}$ & $\begin{array}{c}\text { estándar 10\% } \\
\overline{\mathrm{x}}+\mathrm{EE}\end{array}$ \\
\hline $\mathrm{PC}(\mathrm{g})$ & $2.288 \pm 22,5$ & $2.313 \pm 23,3$ & $2.133 \pm 17,4$ & $2.348 \pm 27,8$ \\
\hline $\mathrm{IC}\left(\mathrm{mm}^{2}\right)$ & $556 \pm 21,8$ & $664 \pm 31,9$ & $831 \pm 43,3$ & $1.051 \pm 50,8$ \\
\hline $\mathrm{ACl}(\mathrm{cm})$ & $23,3 \pm 0,35$ & $24,5 \pm 0,50$ & $21,5 \pm 0,35$ & $24,6 \pm 0,57$ \\
\hline $\mathrm{ACa}(\mathrm{cm})$ & $2,89 \pm 0,05$ & $3,41 \pm 0,07$ & $3,40 \pm 0,06$ & $3,68 \pm 0.09$ \\
\hline $\mathrm{LAb}(\mathrm{cm})$ & $4,35 \pm 0,09$ & $4,83 \pm 0,07$ & $4,81 \pm 0,07$ & $5,09 \pm 0,08$ \\
\hline
\end{tabular}

$\bar{x}$ : media aritmética, EE: error estándar. PC: peso corporal; IC: índice cresta; $\mathrm{ACl}$ : ancho cloaca, $\mathrm{ACa}$ a ancho cadera, LAb: distancia longitudinal.

servándose además una interacción estadísticamente significativa entre los distintos niveles de ambos factores. Dicha interacción se explica porque las aves del genotipo ES*A con alimentación estándar fueron más pesadas, en tanto que, con la alimentación estándar+10, el genotipo E alcanzó mayor peso.

Estos hallazgos coinciden con los de investigadores que reportan la existencia de un marcado crecimiento compensatorio previo a la madurez sexual en gallinas reproductoras severamente restringidas durante la etapa de recría, seguida con mayor oferta de alimento al final de la recría ${ }^{1}$. Otros señalan que un aumento en la asignación de alimento hacia el final de la recría. estimula una maduración sexual más uniforme en el lote debido a que existe una declinación de los requerimientos para crecimiento, junto a un incremento de las demandas para el desarrollo del aparato reproductor e inicio de la producción de huevos ${ }^{10}$.

Entre las variables indicadoras de la madurez sexual (IC, ACa, LAb) existieron diferencias significativas relacionadas con la base genética y el programa de alimentación. Estas variables arrojaron valores superiores para el genotipo $\mathrm{E}$ y para las aves que recibieron mayor asignación de alimento. No obstante, se debe señalar que el $\mathrm{ACl}$ registró una interacción significativa entre los factores.

En las aves alimentadas con el programa estándar, el $\mathrm{ACl}$ arrojó diferencias significativas a favor del genotipo ES*A, mientras que con alimentación estándar +10 ambos grupos genéticos se comportaron en forma similar. Estos resultados coinciden con los reportados por quienes hallaron que las aves sexualmente maduras alcanzan aproximadamente $25 \mathrm{~mm}$ de ancho de cloaca. sugiriendo su medición como método práctico para la evaluación de la madurez sexual ${ }^{12}$

$\mathrm{La}$ variable ancho de cadera (ACa) arrojó diferencias entre los distintos grupos genéticos $(\mathrm{p}=0,01)$, resultados que coinciden con estudios de otros investigadores quienes asignan un firme componente genético a las variables relacionadas con la estructura ósea de la pelvis, con fuertes repercusiones en la fertilidad ${ }^{7}$.

En las Tablas 3 y 4 se vuelcan los resultados obtenidos para la condición corporal en ambos genotipos.
Como se puede observar, la mayor asignación de alimento en la etapa de la recría aumentó la proporción de aves que alcanzaron la $\mathrm{CC} 2$, con resultados que fueron significativos.

Los resultados obtenidos para las mismas variables en estudio en la semana 24 , incluyendo la madurez sexual, se muestran en las Tablas 5 y 6. La ausencia de interacciones entre los factores para la mayoría de las variables (Tabla 6) habilitó a realizar un análisis por separado de los resultados obtenidos.

En la semana 24 el peso corporal no registró diferencias significativas entre ambos genotipos estudiados, en tanto que los caracteres sexuales secundarios ( $\mathrm{IC}$ y $\mathrm{ACl}$ ) y los índices zoométricos relacionados con la esfera reproductiva (ACa y LAb) reflejaron un mayor grado de desarrollo para el genotipo E, lo que pone de manifiesto un diferente grado de integración entre el desarrollo orgánico y el aparato reproductor.

Esto confirma la existencia de relaciones alométricas disímiles entre caracteres sexuales secundarios y el

Tabla 2. Significado de los efectos del análisis de la variancia correspondiente a un diseño completamente aleatorizado con un experimento factorial $2 \times 2$.

\begin{tabular}{lccc}
\hline \multirow{2}{*}{ variables } & \multicolumn{3}{c}{ efectos } \\
\cline { 2 - 4 } & genotipo & nivel alim. & interac. $\mathrm{g}^{*} \mathrm{a}$ \\
\hline $\mathrm{PC}(\mathrm{g})$ & $\mathrm{p}=0,0098$ & $\mathrm{p}<0,0001$ & $\mathrm{p}<0,0001$ \\
$\mathrm{IC}\left(\mathrm{mm}^{2}\right)$ & $\mathrm{p}<0,0001$ & $\mathrm{p}<0,0001$ & $\mathrm{p}=0,148$ \\
$\mathrm{ACl}(\mathrm{cm})$ & $p=0,062$ & $p<0,0001$ & $p=0,037$ \\
$\mathrm{ACa}(\mathrm{cm})$ & $\mathrm{p}<0,0001$ & $p<0,0001$ & $p=0,098$ \\
$\mathrm{LAb}(\mathrm{cm})$ & $p<0,0001$ & $p<0,0001$ & $p=0,225$ \\
\hline
\end{tabular}

alim.: alimentación, interac. (interacción), $g * a$ : genético*alimentación, p: significancia.

Tabla 3. Frecuencias relativas de condición corporal en gallinas Campero INTA del genotipo E según programa de alimentación (expresadas como porcentajes)

\begin{tabular}{cccccc}
\hline genética & alimentación & $\mathrm{CC} 1$ & $\mathrm{CC} 2$ & $\mathrm{CC} 3$ & total \\
\hline $\mathrm{E}$ & estándar+10 & 6,56 & 81,97 & 11,48 & 100 \\
$\mathrm{E}$ & estándar & 34,43 & 62,3 & 3,28 & 100 \\
E & total & 20,49 & 72,13 & 7,38 & 100 \\
\hline
\end{tabular}

Chi-cuadrado Pearson ( $\mathrm{p}=0,0003)$. CC: condición corporal.

Tabla 4. Frecuencias relativas de condición corporal en gallinas Campero INTA del genotipo ES*A según programa de alimentación (expresadas como porcentajes).

\begin{tabular}{cccccc}
\hline genética & alimentación & $\mathrm{CC} 1$ & $\mathrm{CC} 2$ & $\mathrm{CC} 3$ & total \\
\hline ES*A & estándar+10 & 6,9 & 82,76 & 10,34 & 100 \\
ES*A & estándar & 33,93 & 55,36 & 10,71 & 100 \\
ES*A & total & 20,18 & 69,3 & 10,53 & 100 \\
\hline
\end{tabular}

Chi-cuadrado Pearson $(p=0,0012)$. CC: condición corporal. 
Tabla 5. Peso corporal e indicadores zoométricos de madurez sexual en dos genotipos de reproductoras camperas INTA bajo dos regímenes de asignación de nutrientes.

\begin{tabular}{lcccc}
\hline \multirow{2}{*}{ variables } & \multicolumn{2}{c}{ híbridas ES*A } & \multicolumn{2}{c}{ sintéticas E } \\
\cline { 2 - 5 } & $\begin{array}{c}\text { estándar } \\
(\overline{\mathrm{x}} \pm \mathrm{EE})\end{array}$ & $\begin{array}{c}\text { estándar 10\% } \\
(\overline{\mathrm{X}} \pm \mathrm{EE})\end{array}$ & $\begin{array}{c}\text { estándar } \\
(\overline{\mathrm{x}} \pm \mathrm{EE})\end{array}$ & $\begin{array}{c}\text { estándar 10\% } \\
(\overline{\mathrm{x}} \pm \mathrm{EE})\end{array}$ \\
\hline $\mathrm{PC}(\mathrm{g})$ & $2.280 \pm 22,17$ & $2.430 \pm 26,72$ & $2.284 \pm 20,16$ & $2.481 \pm 30,48$ \\
$\mathrm{IC}\left(\mathrm{mm}^{2}\right)$ & $628 \pm 28,58$ & $717 \pm 31,65$ & $1.110 \pm 62,98$ & $1.269 \pm 60,17$ \\
$\mathrm{ACl}(\mathrm{cm})$ & $24,34 \pm 0,47$ & $26,34 \pm 0,46$ & $26,39 \pm 0,53$ & $29,12 \pm 0,45$ \\
$\mathrm{ACa}(\mathrm{cm})$ & $4,49 \pm 0,09$ & $4,97 \pm 0,10$ & $4,57 \pm 0,09$ & $5,38 \pm 0,08$ \\
$\mathrm{LAb}(\mathrm{cm})$ & $5,73 \pm 0,09$ & $5,93 \pm 0,10$ & $5,77 \pm 0,09$ & $6,52 \pm 0,10$ \\
$\mathrm{MS}($ días $)$ & $165 \pm 2,83$ & $156 \pm 2,83$ & $164 \pm 0,00$ & $157 \pm 2,83$ \\
\hline
\end{tabular}

$\overline{\mathbf{x}}$ : media aritmética; EE: error estándar.
Se concluye que diferentes asignaciones de alimento durante las etapas de cría y recría, impactan sobre el peso corporal y los indicadores zoométricos en los genotipos maternos del pollo Campero INTA y sus cruzamientos. Además existen diferencias marcadas en la integración entre peso corporal y desarrollo reproductivo en los diferentes genotipos de gallinas Campero INTA, no obstante el programa de alimentación actúa como modulador de la función reproductiva en este tipo de gallinas, produciendo un marcado adelantamiento a la entrada de la madurez sexual.
Tabla 6. Significado de los efectos del análisis de la variancia correspondiente a un diseño completamente aleatorizado con un experimento factorial $2 \times 2$.

\begin{tabular}{lccc}
\hline & \multicolumn{3}{c}{ efectos } \\
\cline { 2 - 4 } & genotipo & nivel & interacción \\
\hline $\mathrm{PC}(\mathrm{g})$ & $\mathrm{p}=0,274$ & $\mathrm{p}<0,0001$ & $\mathrm{p}<0,360$ \\
$\mathrm{IC}\left(\mathrm{mm}^{2}\right)$ & $\mathrm{p}<0,0001$ & $\mathrm{p}=0,0228$ & $\mathrm{p}=0,487$ \\
$\mathrm{ACl}(\mathrm{cm})$ & $\mathrm{p}<0,0001$ & $\mathrm{p}<0,0001$ & $\mathrm{p}=0,420$ \\
$\mathrm{ACa}(\mathrm{cm})$ & $\mathrm{p}=0,011$ & $\mathrm{p}<0,0001$ & $\mathrm{p}=0,079$ \\
$\mathrm{LAb}(\mathrm{cm})$ & $\mathrm{p}=0,002$ & $\mathrm{p}<0,0001$ & $\mathrm{p}=0,005$ \\
$\mathrm{MS}($ días $)$ & $\mathrm{p}>0,9999$ & $\mathrm{p}=0,0099$ & $\mathrm{p}=0,594$ \\
\hline
\end{tabular}

nivel: nivel de alimentación, interacción: genético-alimentaria, p: significancia.

resto del organismo para ambos genotipos, las cuales se plasman en una expresión diferente desde el punto de vista ontogénico entre la esfera genital y el desarrollo somático.

Por su parte el programa de alimentación tuvo un marcado efecto sobre el peso corporal en ambas poblaciones con un mayor desarrollo de los caracteres sexuales y un adelantamiento de la entrada a la madurez sexual, confirmando que la asignación de nutrientes durante periodos críticos de la recría en las reproductoras pesadas, afecta en forma marcada los eventos anatómicos y fisiológicos que intervienen en la entrada a la madurez sexual ${ }^{3}$.

Sin embargo, el análisis de LAb demostró diferencias significativas entre ambos genotipos y programas de alimentación, observándose una interacción significativa entre los distintos niveles de ambos factores. Dicha interacción se explica porque con la alimentación estándar no hay diferencias entre genotipos mientras que con la alimentación estándar $+10 \%$ las aves pertenecientes al genotipo $\mathrm{E}$ presentaron mayor $\mathrm{LAb}$ que las aves del genotipo ES*A.

Si bien en ambos genotipos hubo un aumento en el valor promedio de la variable con el aumento en la asignación de nutrientes, la respuesta fue proporcionalmente mayor en el caso del genotipo $\mathrm{E}^{12}$.

\section{REFERENCIAS}

1. Brody T, Eitan Y, Soller M, Nir I, Nitsan Z. 1980. Compensatory growth and sexual maturity in broiler females reared under severe food restriction from day of hatching. British Poultry Sci 21: 437-446.

2. Decuypere E, Bruggeman V, Onagbesan $O$, Safi $M$. 1999. Endocrine physiology of reproduction in the female chicken: old wine in new bottles. Int Avian \& Poultry Biol Rev 13: 145-153.

3. Eitan Y, Soller M, Rozenboim I. 1998. Comb size and estrogen levels toward the onset of lay in broiler and layer strain females under ad libitum and restricted feeding. Poultry Sci 77: 1593-1600.

4. Gregory N, Robins J. 1998.A body condition scoring system for layer hens. New Zealand J Agric Res 41: 555-559.

5. Johnson AL. 2000. Reproduction in the female. In: Sturkie's Avian Physiology, $5^{\circ}$ ed., Academics Press, p. 569

6. Joseph NS, Robinson FE, Renema RA, Thorsteinson KA. 2003. Comb growth during sexual maturation in female broiler breeders. J Appl Poult Res 12: 7-13.

7. McGary S, Estevez I, Bakst M. 2003. Potential relationships between physical traits and male broiler breeder fertility. Poultry $S_{c i}$ 82: 328-337.

8. Rauw W, Kanis E, Noordhuizen SE, Grommers FJ. 1998. Undesirable side effects of selection for high production efficiency in farm animals: a review. Livestock Production Sci 56: 15-33.

9. Reddish JM. 2004. Evaluation of the effects of selection for increased body weight and increased yield on growth and development of poultry. Dissertation. Degree Doctor of Philosophy in The Ohio State University, $111 \mathrm{p}$.

10. Renema RA, Rustad ME, Robinson FE. 2007. Implications of changes to commercial broiler and broiler breeder body weight targets over the past 30 years. World's Poultry Sci J 63: 457-472.

11. Steel R, Torrie J. 1988. Bioestadistica: principios y procedimientos, McGraw-Hill/Interamericana, México.

12. Wright D et al. 2012. Onset of sexual maturity in female chickens is genetically linked to loci associated with fecundity and a sexual ornament. Reprod Domestic Anim 47: 31-36. 